\title{
Nephroprotective effect of mesenchymal stem cells in therapy of kidney disease induced by toxicant
}

\section{Tianbiao Zhou ( $\nabla$ zhoutb@aliyun.com )}

Shantou University Medical College https://orcid.org/0000-0001-8065-1644

\section{Shujun Lin}

Shantou University Medical College

\section{Chunling Liao}

Shantou University Medical College

\section{Wenshan Lin}

Shantou University Medical College

Hongzhen Zhong

Shantou University Medical College

\section{Research}

Keywords: Kidney disease, Toxicant, Mesenchymal stem cells, Meta-analysis

Posted Date: May 8th, 2020

DOI: https://doi.org/10.21203/rs.3.rs-25964/v1

License: (c) (1) This work is licensed under a Creative Commons Attribution 4.0 International License. Read Full License 


\section{Abstract \\ Background}

Renal damage caused by drug toxicity is becoming more and more common in clinic. How to avoid and treat kidney damage caused by drug toxicity is essential to maintain patient health and reduce social economic burden. In this study, we performed a meta-analysis to assess the nephroprotective effect of mesenchymal stem cells (MSCs) in therapy of kidney disease induced by toxicant.

\section{Methods}

Cochrane Library, Embase, ISI Web of Science and PubMed databases were searched up to Dec 31, 2019 to identify the studies and extract the data to assess the efficacy of MSCs for kidney disease induced by toxicant using Cochrane Review Manager Version 5.3.

\section{Results}

27 studies were eligible and recruited for this meta-analysis. The results showed that the difference of Scr between MSCs treatment group and control group was notable for 2 days, 4 days, 5 days, $6-8$ days, 1015 days, $\geq 42$ days ( 2 days: $W M D=-0.88,95 \% C l:-1.34,-0.42, P=0.0002 ; 4$ days: $W M D=-0.69,95 \% \mathrm{Cl}$ : $-0.99,-0.39, \mathrm{P}<0.00001 ; 5$ days: $\mathrm{WMD}=-0.46,95 \% \mathrm{Cl}:-0.67,-0.25, \mathrm{P}<0.0001 ; 6-8$ days: $\mathrm{WMD}=-0.51$, $95 \% \mathrm{Cl}:-0.79,-0.22, \mathrm{P}=0.0005 ; 10-15$ days: $\mathrm{WMD}=-0.38,95 \% \mathrm{Cl}:-0.56,-0.20, \mathrm{P}<0.0001 ; \geq 42$ days: WMD $=-0.22,95 \% \mathrm{Cl}:-0.39,-0.06, \mathrm{P}=0.007)$. Furthermore, the difference of BUN between MSCs treatment group and control group was notable for $2-3$ days, $4-5$ days, $6-8$ days, $\geq 28$ days. The results also indicated that MSCs treatment can alleviate the inflammatory cells, necrotic tubule, regenerative tubules, renal interstitial fibrosis in kidney disease induced by toxicant.

\section{Conclusion}

MSCs might be a promising therapeutic agent for kidney disease induced by toxicant.

\section{Background}

Kidney injury includes acute kidney injury and chronic kidney disease and it is a common condition to be associated with the morbidity and mortality of patients. When the acute kidney injury is not alleviated in time, it will develop into chronic kidney disease. Toxicant-induced kidney injury is one of the most common causes for kidney disease, and it also causes substantial morbidity and retards drug development [1]. At present, renal damage caused by drug toxicity is becoming more and more common in clinic. How to avoid and treat kidney damage caused by drug toxicity is essential to maintain patient health and reduce social economic burden. 
Mesenchymal stem cells (MSCs), being pluripotent mesenchymal cells present in various tissues with self-regeneration, have multilineage differentiation ability under an appropriate environment, and are easy to obtain; therefore, they are a promising therapeutic option for some diseases due to their unique properties of releasing some important bioactive factors [2-4]. Drug toxicity results in renal tubular epithelial cell damage or death, and can lead to renal interstitial inflammation which develops into renal interstitial fibrosis and renal loss. In previous, some studies found that MSCs can play a protective role against the injury of renal tubular epithelial cell and prevent the renal interstitial fibrosis[5-9]. In this study, we performed a meta-analysis to assess the nephroprotective effect of MSCs in therapy of kidney disease induced by toxicant.

\section{Materials And Methods Search strategy}

We searched databases (Cochrane Library, Embase, ISI Web of Science and PubMed databases) up to Dec 31, 2019, for the following search corresponding terms: (mesenchymal stem cells OR MSC OR MSCs OR multipotent stromal cells OR mesenchymal stromal cells OR mesenchymal progenitor cells OR stem cells) AND (gentamicin OR aristolochic acid OR cisplatin OR adriamycin OR cadmium chloride OR methotrexate OR rifampicin OR glycerol OR streptozocin) AND (kidney injury OR renal failure OR kidney disease), only confined to English-language literature. An additional search was conducted among the eligible manual references of the cited articles.

\section{Inclusion And Exclusion Criteria Inclusion criteria:}

Our meta-analysis includes studies analyzing the efficacy of MSCs treatment on the mice or rat with kidney disease.

\section{Exclusion criteria}

(1) letters, case reports, reviews, clinical studies, editorials, meta-analysis and systematic reviews; (2) studies lacked the targeted indicators or number of case group or control group, and were conducted in humans; (3) the kidney disease was not induced by toxicant, and (4) the therapeutic regimen for kidney disease including other agents with undefined effects.

\section{Outcome Measures}

We filter the following outcomes associated with the efficacy of MSCs treatment from the recruited studies: serum creatinine (Scr), blood urea nitrogen (BUN), urinary albumin excretion (UAE), 
malondialdehyde (MDA), L-Glutathione (GSH), CAT, superoxide dismutase (SOD), and renal pathology. Also, we conducted a mutual consensus when met with disagreements.

\section{Quality Assessment}

Two investigators evaluate the methodological quality with The Cochrane Handbook for Interventions independently. We assessed the following sections of every investigation: selection bias, attrition bias, performance bias, detection bias, reporting bias, and other bias. Each item was classified as unclear, high risk or low risk.

\section{Statistical analysis}

Review Manager Version 5.3 was applied to explore whether MSCs treatment can acquire a good efficacy on kidney disease induced by toxicant and STATA 12.0 were used to test the publication bias. Heterogeneity of variation among individual studies was quantified and described with the $\mathrm{I}^{2}$. The fixed effect model was used if the $p$-value of the the heterogeneity test was $\geq 0.1$. Otherwise, the random effects model will be applied to pool the outcomes. Besides, to compute the continuous variables, we analyze weighted mean differences (WMDs) for the mean values. We also calculated $95 \%$ confidence intervals $(95 \% \mathrm{Cl})$ for the included studies using the Mantel-Haenszel $(\mathrm{M}-\mathrm{H})$ method. Additionally, we evaluate the publication bias with Begg's rank correlation test as well as Egger's linear regression method among the studies. A $p$-value $<0.05$ was considered as statistical significance.

\section{Results}

\section{Search results}

The databases mentioned above were searched for this meta-analysis, and we only recruited these studies in mice or rat for evaluation of therapeutic efficiency of MSCs treatment on kidney disease induced by toxicant. Twenty studies [10-36] were eligible and recruited for this meta-analysis, and the flowchart of inclusion of studies is presented in Fig. 1. The included study characteristics are shown in Table 1. 
Table 1

Characteristics of the studies included in this meta-analysis.

\begin{tabular}{|c|c|c|c|c|c|c|c|}
\hline Author, year & $\mathrm{n}$ & $\begin{array}{l}\text { Type } \\
\text { of } \\
\text { animal }\end{array}$ & Type of injury & $\begin{array}{l}\text { MSC } \\
\text { type }\end{array}$ & $\begin{array}{l}\text { Number } \\
\text { of MSC }\end{array}$ & $\begin{array}{l}\text { Route of } \\
\text { delivery }\end{array}$ & $\begin{array}{l}\text { Endpoints } \\
\text { for this } \\
\text { meta- } \\
\text { analysis }\end{array}$ \\
\hline $\begin{array}{l}\text { Herrera } \\
2004\end{array}$ & 24 & Mice & $\begin{array}{l}\text { Glycerol- } \\
\text { induced }\end{array}$ & $\begin{array}{l}\text { BM- } \\
\text { MSCs }\end{array}$ & $1 \times 10^{6}$ & $\begin{array}{l}\text { Intravenous } \\
\text { injection }\end{array}$ & Scr \\
\hline Bi 2007 & 12 & Mice & $\begin{array}{l}\text { Cisplatin- } \\
\text { Induced }\end{array}$ & $\begin{array}{l}\text { BM- } \\
\text { MSCs }\end{array}$ & $2 \times 10^{5}$ & $\begin{array}{l}\text { Intravenous } \\
\text { injection or } \\
\text { intraperitoneal } \\
\text { injection }\end{array}$ & Scr, BUN, \\
\hline Sun 2008 & 40 & Rat & $\begin{array}{l}\text { Glycerol- } \\
\text { induced }\end{array}$ & $\begin{array}{l}\text { BM- } \\
\text { MSCs }\end{array}$ & $2 \times 10^{6}$ & $\begin{array}{l}\text { Abdominal } \\
\text { aorta injection }\end{array}$ & Scr, BUN, \\
\hline Qian 2008 & 6 & Rat & $\begin{array}{l}\text { Glycerol- } \\
\text { induced }\end{array}$ & $\begin{array}{l}\text { BM- } \\
\text { MSCs }\end{array}$ & $1 \times 10^{4}$ & $\begin{array}{l}\text { Intravenous } \\
\text { injection }\end{array}$ & Scr \\
\hline $\begin{array}{l}\text { Magnasco } \\
2008\end{array}$ & 22 & Rat & $\begin{array}{l}\text { Adriamycin- } \\
\text { induced }\end{array}$ & $\begin{array}{l}\text { BM- } \\
\text { MSCs }\end{array}$ & $3 \times 10^{6}$ & $\begin{array}{l}\text { Intravenous } \\
\text { injection }\end{array}$ & $\begin{array}{l}\text { Scr, BUN, } \\
\text { UAE, renal } \\
\text { damage } \\
\text { score }\end{array}$ \\
\hline Bruno 2009 & 16 & Mice & $\begin{array}{l}\text { Glycerol- } \\
\text { induced }\end{array}$ & $\begin{array}{l}\text { BM- } \\
\text { MSCs }\end{array}$ & - & $\begin{array}{l}\text { Intravenous } \\
\text { injection }\end{array}$ & $\begin{array}{l}\text { Scr, BUN, } \\
\text { MDA, GSH, } \\
\text { SOD, renal } \\
\text { damage } \\
\text { score }\end{array}$ \\
\hline $\begin{array}{l}\text { Eliopoulos } \\
2010\end{array}$ & 10 & Mice & $\begin{array}{l}\text { Cisplatin- } \\
\text { induced }\end{array}$ & $\begin{array}{l}\text { BM- } \\
\text { MSCs }\end{array}$ & $5 \times 10^{6}$ & $\begin{array}{l}\text { Intraperitoneal } \\
\text { injection }\end{array}$ & Scr, BUN \\
\hline Kim 2012 & 17 & Rat & $\begin{array}{l}\text { Cisplatin- } \\
\text { induced }\end{array}$ & $\begin{array}{l}\text { AD- } \\
\text { MSCs }\end{array}$ & $5 \times 10^{5}$ & $\begin{array}{l}\text { Intravenous } \\
\text { injection }\end{array}$ & Scr, BUN \\
\hline Zickri 2012 & 30 & Rat & $\begin{array}{l}\text { Adriamycin- } \\
\text { induced }\end{array}$ & $\begin{array}{l}\text { hUC- } \\
\text { MSCs }\end{array}$ & $5 \times 10^{5}$ & $\begin{array}{l}\text { Intravenous } \\
\text { injection }\end{array}$ & Scr \\
\hline $\begin{array}{l}\text { Sarhan } \\
2014\end{array}$ & 19 & Rat & $\begin{array}{l}\text { Adriamycin- } \\
\text { induced }\end{array}$ & $\begin{array}{l}\text { BM- } \\
\text { MSCs }\end{array}$ & $4 \times 10^{6}$ & $\begin{array}{l}\text { Intravenous } \\
\text { injection }\end{array}$ & $\begin{array}{l}\text { Scr, BUN, } \\
\text { UAE, renal } \\
\text { pathology, } \\
\text { MDA, GSH }\end{array}$ \\
\hline $\begin{array}{l}\text { Moustafa } \\
2016\end{array}$ & 80 & Rat & $\begin{array}{l}\text { Cisplatin- } \\
\text { induced }\end{array}$ & $\begin{array}{l}\text { BM- } \\
\text { MSCs }\end{array}$ & $5 \times 10^{6}$ & $\begin{array}{l}\text { Intravenous, } \\
\text { intra-arterial or } \\
\text { kidney sub } \\
\text { capsular } \\
\text { injection }\end{array}$ & $\begin{array}{l}\text { Scr, MDA, } \\
\text { GSH, SOD }\end{array}$ \\
\hline \multicolumn{8}{|c|}{$\begin{array}{l}\text { Note: BM-MSCs: bone marrow mesenchymal stem cells; hAMSCs: human amnion-derived } \\
\text { mesenchymal stem cells; hUC-MSCs: human umbilical cord-mesenchymal stem cells; AD-MSCs: } \\
\text { adipose tissue-derived mesenchymal stem cells; mESCs: mouse embryonic stem cells; AFSCs: } \\
\text { amniotic fluid stem cells; USCs: urine-derived stem cells; Scr: serum creatinine; BUN: blood urea } \\
\text { nitrogen; UAE: urinary albumin excretion; Ccr: creatinine clearance rate; MDA: malondialdehyde; GSH: } \\
\text { L-Glutathione; SOD: superoxide dismutase. }\end{array}$} \\
\hline
\end{tabular}




\begin{tabular}{|c|c|c|c|c|c|c|c|}
\hline Author, year & $\mathrm{n}$ & $\begin{array}{l}\text { Type } \\
\text { of } \\
\text { animal }\end{array}$ & Type of injury & $\begin{array}{l}\text { MSC } \\
\text { type }\end{array}$ & $\begin{array}{l}\text { Number } \\
\text { of MSC }\end{array}$ & $\begin{array}{l}\text { Route of } \\
\text { delivery }\end{array}$ & $\begin{array}{l}\text { Endpoints } \\
\text { for this } \\
\text { meta- } \\
\text { analysis }\end{array}$ \\
\hline $\begin{array}{l}\text { Elhusseini } \\
2016\end{array}$ & 40 & Rat & $\begin{array}{l}\text { Cisplatin- } \\
\text { induced }\end{array}$ & $\begin{array}{l}\text { AD- } \\
\text { MSCs }\end{array}$ & $5 \times 10^{6}$ & $\begin{array}{l}\text { Intravenous } \\
\text { injection }\end{array}$ & $\begin{array}{l}\text { Ccr, renal } \\
\text { pathology, } \\
\text { MDA, GSH, } \\
\text { SOD }\end{array}$ \\
\hline Anan 2016 & 13 & Rat & $\begin{array}{l}\text { Adriamycin- } \\
\text { induced }\end{array}$ & $\begin{array}{l}\text { BM- } \\
\text { MSCs }\end{array}$ & $1 \times 10^{6}$ & $\begin{array}{l}\text { Intravenous } \\
\text { injection }\end{array}$ & $\begin{array}{l}\text { Scr, BUN, } \\
\text { SOD }\end{array}$ \\
\hline Gad 2017 & 24 & Rat & $\begin{array}{l}\text { Methotrexate- } \\
\text { induced }\end{array}$ & $\begin{array}{l}\text { BM- } \\
\text { MSCs }\end{array}$ & $2 \times 10^{6}$ & $\begin{array}{l}\text { Intraperitoneal } \\
\text { injection }\end{array}$ & $\begin{array}{l}\text { Scr, BUN, } \\
\text { MDA, GSH }\end{array}$ \\
\hline $\begin{array}{l}\text { Rashed } \\
2018\end{array}$ & 20 & Rat & $\begin{array}{l}\text { Streptozotocin } \\
\text {-induced }\end{array}$ & $\begin{array}{l}\text { BM- } \\
\text { MSCs }\end{array}$ & $1 \times 10^{6}$ & $\begin{array}{l}\text { Intravenous } \\
\text { injection }\end{array}$ & $\begin{array}{l}\text { Scr, BUN, } \\
\text { UAE, Ccr }\end{array}$ \\
\hline $\begin{array}{l}\text { Elbaghdady } \\
2018\end{array}$ & 20 & Rat & $\begin{array}{l}\text { Cadmium } \\
\text { chloride- } \\
\text { induced }\end{array}$ & $\begin{array}{l}\text { BM- } \\
\text { MSCs }\end{array}$ & $2 \times 10^{6}$ & $\begin{array}{l}\text { Intravenous } \\
\text { injection }\end{array}$ & Scr \\
\hline $\begin{array}{l}\text { Danjuma } \\
2018\end{array}$ & 16 & Rat & $\begin{array}{l}\text { Rifampicin- } \\
\text { induced }\end{array}$ & $\begin{array}{l}\text { BM- } \\
\text { MSCs }\end{array}$ & $\begin{array}{l}2.5 \times \\
10 \rrbracket\end{array}$ & $\begin{array}{l}\text { Intravenous } \\
\text { injection }\end{array}$ & Scr, BUN \\
\hline Putra 2019 & 10 & Rat & $\begin{array}{l}\text { Gentamicin } \\
\text { induced }\end{array}$ & $\begin{array}{l}\text { hUC- } \\
\text { MSCs }\end{array}$ & $1 \times 10^{6}$ & $\begin{array}{l}\text { Intraperitoneal } \\
\text { injection }\end{array}$ & $\begin{array}{l}\text { Scr, BUN, } \\
\text { renal } \\
\text { pathology }\end{array}$ \\
\hline $\begin{array}{l}\text { Cetinkaya } \\
2019\end{array}$ & 17 & Rat & $\begin{array}{l}\text { Aristolochic } \\
\text { acid induced }\end{array}$ & hAMSC & 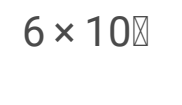 & $\begin{array}{l}\text { Intravenous } \\
\text { injection }\end{array}$ & Scr, BUN \\
\hline Selim 2019 & 70 & Rat & $\begin{array}{l}\text { Cisplatin- } \\
\text { induced }\end{array}$ & $\begin{array}{l}\text { AD- } \\
\text { MSCs; } \\
\text { BM- } \\
\text { MSCs }\end{array}$ & $4 \times 10^{6}$ & $\begin{array}{l}\text { Intravenous } \\
\text { injection }\end{array}$ & Scr, BUN \\
\hline $\begin{array}{l}\text { Mata- } \\
\text { Miranda } \\
2019\end{array}$ & 10 & Mice & $\begin{array}{l}\text { Cisplatin- } \\
\text { induced }\end{array}$ & mESCs & $1 \times 10^{6}$ & $\begin{array}{l}\text { Intraperitoneal } \\
\text { injection }\end{array}$ & Scr \\
\hline $\begin{array}{l}\text { Vazquez- } \\
\text { Zapien } \\
2019\end{array}$ & 19 & Mice & $\begin{array}{l}\text { Cisplatin- } \\
\text { induced }\end{array}$ & mESCs & $1 \times 10^{6}$ & $\begin{array}{l}\text { Intraperitoneal } \\
\text { injection }\end{array}$ & Scr \\
\hline $\begin{array}{l}\text { Minocha } \\
2019\end{array}$ & 3 & Rat & $\begin{array}{l}\text { Cisplatin- } \\
\text { induced }\end{array}$ & AFSC & $2 \times 10^{6}$ & $\begin{array}{l}\text { Intravenous } \\
\text { injection }\end{array}$ & Scr, BUN \\
\hline Sun B 2019 & 10 & Rat & $\begin{array}{l}\text { Cisplatin- } \\
\text { induced }\end{array}$ & USCs & $2 \times 10^{6}$ & $\begin{array}{l}\text { Intravenous } \\
\text { injection }\end{array}$ & Scr \\
\hline \multicolumn{8}{|c|}{$\begin{array}{l}\text { Note: BM-MSCs: bone marrow mesenchymal stem cells; hAMSCs: human amnion-derived } \\
\text { mesenchymal stem cells; hUC-MSCs: human umbilical cord-mesenchymal stem cells; AD-MSCs: } \\
\text { adipose tissue-derived mesenchymal stem cells; mESCs: mouse embryonic stem cells; AFSCs: } \\
\text { amniotic fluid stem cells; USCs: urine-derived stem cells; Scr: serum creatinine; BUN: blood urea } \\
\text { nitrogen; UAE: urinary albumin excretion; Ccr: creatinine clearance rate; MDA: malondialdehyde; GSH: } \\
\text { L-Glutathione; SOD: superoxide dismutase. }\end{array}$} \\
\hline
\end{tabular}




\begin{tabular}{|c|c|c|c|c|c|c|c|}
\hline Author, year & $\mathrm{n}$ & $\begin{array}{l}\text { Type } \\
\text { of } \\
\text { animal }\end{array}$ & Type of injury & $\begin{array}{l}\text { MSC } \\
\text { type }\end{array}$ & $\begin{array}{l}\text { Number } \\
\text { of MSC }\end{array}$ & $\begin{array}{l}\text { Route of } \\
\text { delivery }\end{array}$ & $\begin{array}{l}\text { Endpoints } \\
\text { for this } \\
\text { meta- } \\
\text { analysis }\end{array}$ \\
\hline Sun 2019 & 6 & Rat & $\begin{array}{l}\text { Cisplatin- } \\
\text { induced }\end{array}$ & $\begin{array}{l}\text { BM- } \\
\text { MSCs }\end{array}$ & - & $\begin{array}{l}\text { Renal } \\
\text { parenchyma } \\
\text { injection }\end{array}$ & Scr, BUN \\
\hline $\begin{array}{l}\text { Zhang } \\
2020\end{array}$ & 9 & Rat & $\begin{array}{l}\text { Cisplatin- } \\
\text { induced }\end{array}$ & USCs & $5 \times 10^{6}$ & $\begin{array}{l}\text { Subcutaneous } \\
\text { injection }\end{array}$ & $\begin{array}{l}\text { Scr, Ccr, } \\
\text { renal } \\
\text { pathology }\end{array}$ \\
\hline $\begin{array}{l}\text { Foroutan } \\
2020\end{array}$ & 6 & Rat & $\begin{array}{l}\text { Cisplatin- } \\
\text { induced }\end{array}$ & $\begin{array}{l}\text { BM- } \\
\text { MSCs }\end{array}$ & - & $\begin{array}{l}\text { Intraperitoneal } \\
\text { injection }\end{array}$ & Scr, BUN \\
\hline \multicolumn{8}{|c|}{$\begin{array}{l}\text { Note: BM-MSCs: bone marrow mesenchymal stem cells; hAMSCs: human amnion-derived } \\
\text { mesenchymal stem cells; hUC-MSCs: human umbilical cord-mesenchymal stem cells; AD-MSCs: } \\
\text { adipose tissue-derived mesenchymal stem cells; mESCs: mouse embryonic stem cells; AFSCs: } \\
\text { amniotic fluid stem cells; USCs: urine-derived stem cells; Scr: serum creatinine; BUN: blood urea } \\
\text { nitrogen; UAE: urinary albumin excretion; Ccr: creatinine clearance rate; MDA: malondialdehyde; GSH: } \\
\text { L-Glutathione; SOD: superoxide dismutase. }\end{array}$} \\
\hline
\end{tabular}

\section{Quality Assessment Of Included Studies}

In the recruited studies, the methodological quality was considered as acceptable, for the result that most of the domains of the recruited investigations were ranked as unclear risk of bias or low risk of bias. Unclear risk of bias mostly detected in performance bias and selection bias. Low risk of bias was mostly occurred in detection bias, reporting bias, and attrition bias. Figure 2 showed the summary of the risk of biases of the recruited investigations.

\section{Scr}

26 studies [10-15, 17-36] was included to assess the effect of MSCs on Scr, three for 2 days, four for 3day, five for 4 days, six for 5-day, seven for 6-8 days, ten for 10-15 days, six for 28-30 days, and six for $\geq 42$ days, and the results showed that the difference between MSCs treatment group and control group was notable for 2 days, 4 days, 5 days, $6-8$ days, $10-15$ days, $\geq 42$ days ( 2 days: $W M D=-0.88,95 \% \mathrm{Cl}$ : $-1.34,-0.42, \mathrm{P}=0.0002 ; 4$ days: $\mathrm{WMD}=-0.69,95 \% \mathrm{Cl}:-0.99,-0.39, \mathrm{P}<0.00001 ; 5$ days: $\mathrm{WMD}=-0.46,95 \% \mathrm{Cl}$ : $-0.67,-0.25, \mathrm{P}<0.0001 ; 6-8$ days: $\mathrm{WMD}=-0.51,95 \% \mathrm{Cl}:-0.79,-0.22, \mathrm{P}=0.0005 ; 10-15$ days: $\mathrm{WMD}=-0.38$, $95 \% \mathrm{Cl}:-0.56,-0.20, \mathrm{P}<0.0001 ; \geq 42$ days: $\mathrm{WMD}=-0.22,95 \% \mathrm{Cl}:-0.39,-0.06, \mathrm{P}=0.007$; Fig. 3 and Table 2). However, the difference between MSCs treatment group and control group was not notable for 3 days and $28-30$ days ( 3 days: $W M D=-0.09,95 \% C l:-0.25,-0.06, P=0.24 ; 28-30$ days: $W M D=-0.59,95 \% C l:-1.22$, $-0.05, \mathrm{P}=0.07$; Fig. 3 and Table 2). 
Table 2

Meta-analysis of the efficacy of MSC in therapy of renal injury induced by toxicant

\begin{tabular}{|c|c|c|c|c|c|c|}
\hline Indicators & $\begin{array}{l}\text { Time } \\
\text { point }\end{array}$ & Studies & $\mathrm{Q}$ test & Model & OR/WMD & $\mathbf{P}$ \\
\hline & & Number & $P$-value & selected & $(95 \% \mathrm{Cl})$ & \\
\hline \multirow[t]{8}{*}{ Scr } & 2 days & 3 & 0.001 & Random & $\begin{array}{l}-0.88(-1.34 \\
-0.42)\end{array}$ & 0.0002 \\
\hline & 3 days & 4 & 0.0004 & Random & $-0.09(-0.25,0.06)$ & 0.24 \\
\hline & 4 days & 5 & 0.0002 & Random & $\begin{array}{l}-0.69(-0.99 \\
-0.39)\end{array}$ & $<.00001$ \\
\hline & 5 days & 6 & $<.00001$ & Random & $\begin{array}{l}-0.46(-0.67 \\
-0.25)\end{array}$ & $\begin{array}{l}< \\
0.0001\end{array}$ \\
\hline & $6-8$ days & 6 & $<.00001$ & Random & $\begin{array}{l}-0.51(-0.79 \\
-0.22)\end{array}$ & 0.0005 \\
\hline & $\begin{array}{l}10-15 \\
\text { days }\end{array}$ & 10 & $\begin{array}{l}<.00001 \\
0 .\end{array}$ & Random & $\begin{array}{l}-0.38(-0.56, \\
-0.20)\end{array}$ & $\begin{array}{l}<.0001 \\
0.0\end{array}$ \\
\hline & $\begin{array}{l}28-30 \\
\text { days }\end{array}$ & 6 & $\begin{array}{l}< \\
0.00001\end{array}$ & Random & $-0.59(-1.22,0.05)$ & 0.07 \\
\hline & $\begin{array}{l}\geq 42 \\
\text { days }\end{array}$ & 6 & $\begin{array}{l}<.00001 \\
0\end{array}$ & Random & $\begin{array}{l}-0.22(-0.39 \\
-0.06)\end{array}$ & 0.007 \\
\hline \multirow[t]{5}{*}{ BUN } & $2-3$ days & 6 & $<.00001$ & Random & $\begin{array}{l}-25.08(-37.49 \\
-12.67)\end{array}$ & $\begin{array}{l}< \\
0.0001\end{array}$ \\
\hline & $4-5$ days & 7 & $\begin{array}{l}<.00001 \\
0.0\end{array}$ & Random & $\begin{array}{l}-24.37(-31.66, \\
-17.07)\end{array}$ & $\begin{array}{l}<.00001 \\
0 .\end{array}$ \\
\hline & $6-8$ days & 4 & $\begin{array}{l}<.00001 \\
0.0\end{array}$ & Random & $\begin{array}{l}-33.44(-59.37 \\
-7.51)\end{array}$ & 0.01 \\
\hline & $\begin{array}{l}13-15 \\
\text { days }\end{array}$ & 4 & $\begin{array}{l}<.00001 \\
\end{array}$ & Random & $\begin{array}{l}-13.40(-32.34 \\
5.54)\end{array}$ & 0.17 \\
\hline & $\begin{array}{l}\geq 28 \\
\text { days }\end{array}$ & 7 & $<.00001$ & Random & $\begin{array}{l}-19.85(-33.35 \\
-6.35)\end{array}$ & 0.004 \\
\hline UAE & - & 3 & 0.72 & Fixed & $\begin{array}{l}-22.66(-26.41 \\
-18.90)\end{array}$ & $\begin{array}{l}<.00001 \\
0 .\end{array}$ \\
\hline MDA & - & 4 & 0.41 & Fixed & $\begin{array}{l}-17.21(-20.38, \\
-14.04)\end{array}$ & $\begin{array}{l}< \\
0.00001\end{array}$ \\
\hline GSH & - & 4 & $<.00001$ & Random & $4.62(2.74,6.50)$ & $<.00001$ \\
\hline
\end{tabular}

Note: Scr: serum creatinine; BUN: blood urea nitrogen; UAE: urinary albumin excretion; Ccr: creatinine clearance rate; MDA: malondialdehyde; GSH: L-Glutathione; SOD: superoxide dismutase. 


\begin{tabular}{|c|c|c|c|c|c|c|}
\hline Indicators & $\begin{array}{l}\text { Time } \\
\text { point }\end{array}$ & Studies & Q test & Model & OR/WMD & $\mathbf{P}$ \\
\hline SOD & - & 3 & $<.00001$ & Random & $5.42(2.92,7.93)$ & $\begin{array}{l}< \\
0.0001\end{array}$ \\
\hline \multicolumn{7}{|l|}{ Renal pathology } \\
\hline Inflammatory cells & - & 4 & $<.00001$ & Random & $\begin{array}{l}-2.66(-3.83, \\
-1.49)\end{array}$ & $\begin{array}{l}< \\
0.00001\end{array}$ \\
\hline Necrotic tubule & - & 2 & $\begin{array}{l}<.00001 \\
0 .\end{array}$ & Random & $\begin{array}{l}-2.58(-4.75 \\
-0.40)\end{array}$ & 0.02 \\
\hline $\begin{array}{l}\text { Regenerative } \\
\text { tubules }\end{array}$ & - & 2 & - & Fixed & $6.00(3.45,8.55)$ & $\begin{array}{l}< \\
0.00001\end{array}$ \\
\hline $\begin{array}{l}\text { Renal interstitial } \\
\text { fibrosis }\end{array}$ & - & 3 & $<.00001$ & Random & $\begin{array}{l}-5.82(-7.41 \\
-4.23)\end{array}$ & $<.00001$ \\
\hline
\end{tabular}

\section{BUN}

17 studies $[10-14,17,18,20,21,23,25-28,31-33,35,36]$ was included to assess the effect of MSCs on BUN, six for 2-3 days, seven for 4-5 days, four for 6-8 days, four for 13-15 days, and seven for $\geq 28$ days, and the results indicated that the difference between MSCs treatment group and control group was notable for $2-3$ days, $4-5$ days, $6-8$ days, $\geq 28$ days ( $2-3$ days: $W M D=-25.08,95 \% C l:-37.49,-12.67, \mathrm{P}<$ $0.0001 ; 4-5$ days: $\mathrm{WMD}=-24.37,95 \% \mathrm{Cl}:-31.66,-17.07, \mathrm{P}<0.00001 ; 6-8$ days: $\mathrm{WMD}=-33.44,95 \% \mathrm{Cl}$ : $-59.37,-7.51, \mathrm{P}=0.01 ; \geq 28$ days: $\mathrm{WMD}=-19.85,95 \% \mathrm{Cl}:-33.35,-6.35, \mathrm{P}=0.004 ;$ Fig. 4 and Table 2 ). However, the difference between MSCs treatment group and control group was not notable for 13-15 days (WMD=-13.40, 95\%Cl: $-32.34,5.54, \mathrm{P}=0.17$; Fig. 4 and Table 2).

\section{Urinary Albumin Excretion}

Three studies $[21,25,26]$ were recruited into the meta-analysis for the assessment of MSCs on UAE. The results showed that the MSCs group had lower UAE than the control group (WMD $=-22.66,95 \% \mathrm{Cl}:-26.41$, $-18.90, \mathrm{P}<0.00001$; Table 2).

\section{Oxidative Stress}

In this meta-analysis, four studies $[16,18,22,26]$ were included for the assessment of MDA, four $[16,18$, $22,26]$ for GSH, three $[10,16,22]$ for SOD. The results indicated that the difference between MSCs treatment group and control group was notable for MDA, GSH, SOD (MDA: WMD=-17.21, 95\%Cl: -20.38, 
-14.04, P< 0.00001; GSH: WMD = 4.62, 95\%Cl: 2.74, 6.50, P< 0.00001; SOD: WMD = 5.42, 95\%Cl: 2.92, 7.93, $\mathrm{P}<0.0001 ;$ Table 2).

\section{Assessment Of Renal Pathology}

Four studies $[16,23,26,34]$ for inflammatory cells, two studies $[16,26]$ for necrotic tubule, two studies $[16,26]$ for regenerative tubules and three studies $[16,26,34]$ for renal interstitial fibrosis were included into this meta-analysis. The results indicated that the difference of inflammatory cells, necrotic tubule, regenerative tubules, renal interstitial fibrosis between MSCs treatment and control group was significant (inflammatory cells: $\mathrm{WMD}=-2.66,95 \% \mathrm{Cl}:-3.83,-1.49, \mathrm{P}<0.00001$; necrotic tubule: $\mathrm{WMD}=-2.58,95 \% \mathrm{Cl}$ : $-4.75,-0.40, P=0.02$; regenerative tubules: $\mathrm{WMD}=6.00,95 \% \mathrm{Cl}: 3.45,8.55, \mathrm{P}<0.00001$; renal interstitial fibrosis: $W M D=-5.82,95 \% \mathrm{Cl}:-7.41,-4.23, \mathrm{P}<0.00001$; Table 2).

\section{Publication bias}

The publication bias was tested in this meta-analysis, and a funnel plot generated used STATA 12.0 for the primary outcome, and Begg'stest and Egger's test suggested that publication bias was found (Egger's: $P=0.000$, Begg's: $P=0.000 ;$ Fig. 5).

\section{Discussion}

We reviewed all the included studies and included the various of Scr, BUN, urinary albumin excretion, oxidative stress, renal pathology to assess the nephroprotective effect of MSCs in therapy of kidney disease induced by toxicant. We found that MSCs treatment can reduce the Scr levels at 2 days, 4 days, 5 days, 6-8 days, 10-15 days, $\geq 42$ days in animal models of kidney disease induced by toxicant. Furthermore, MSCs treatment also can reduce the levels of BUN at 2-3 days, $4-5$ days, $6-8$ days, $\geq 28$ days. We also found that MSCs group had lower UAE than the control group. In previous, MSCs treatment can reduce the levels of Scr, BUN, proteinuria in lupus nephritis in mice [37]. Chen et al [38] found that MSCs have ameliorated ischemia/reperfusion injury-induced acute kidney injury in rats and can reduce the Scr levels. Xiu et al [39] found that MSCs transplantation can significantly download the concentration of BUN and SCr, and prevent the event of the tissue injury, and reduced mortality after lipopolysaccaride -induced acute kidney injury.

MSCs treatment group got a higher level of GSH, SOD, and a lower level of MDA when compared with control group. El-Metwaly et al [40] found that MSCs can increase the GSH level and reduce the MDA level in lung tissue of acute lung injury rats. Zhang et al [41] reported that MSC effectively reduced the level of MDA, and increased SOD in the lung tissue of acute lung injury rats. Liu et al [42] reported that MSC significantly increased the activity of glutathione (GSH), and reduced the levels of MDA in rats induced by unilateral ureteral obstruction. 
Furthermore, our study indicated that the MSCs treatment can alleviate the inflammatory cells, necrotic tubule, regenerative tubules, renal interstitial fibrosis in kidney disease induced by toxicant. In previous, there were some studies indicated that MSCs treatment can alleviate the renal pathological changes in unilateral ureteral obstruction rat or mice $[8,9,43]$.

However, some limitations were also found in this meta-analysis. First, the small sample size was found for the recruited studies. The dose of MSCs administered and the type of MSCs were not exactly same. Publication bias was found in this meta-analysis, and the results should be re-assessed in the future. Furthermore, there were different animal types (mouse and rat). These limitations mentioned above may affect the robust of our results.

\section{Conclusions}

The MSCs treatment can reduce the Scr levels at 2 days, 4 days, 5 days, $6-8$ days, $10-15$ days, $\geq 42$ days, and reduce the BUN levels at $2-3$ days, $4-5$ days, $6-8$ days, $\geq 28$ days. The results also indicated that MSCs treatment can alleviate the inflammatory cells, necrotic tubule, regenerative tubules, renal interstitial fibrosis in kidney disease induced by toxicant.

\section{Abbreviations}

MSCs: mesenchymal stem cells; Scr: serum creatinine; BUN: blood urea nitrogen; UAE: urinary albumin excretion; MDA: malondialdehyde; GSH: L-Glutathione; SOD: superoxide dismutase; WMDs: Weighted mean differences; $\mathrm{Cl}$ : confidence intervals; $\mathrm{M}-\mathrm{H}$ : Mantel-Haenszel.

\section{Declarations}

\section{Acknowledgements}

The authors would like to gratefully acknowledge the most helpful comments on this paper received from Professor Hong Qian, Karolinska Institutet.

\section{Funding}

This study was supported by the Guangzhou Medical Key Discipline Construction Project (2017-2019), the Natural Science Foundation of the Guangdong Province (no. 2015A030310386) and Guangdong Medical Science and Technology Research Fund Project (no. A2018336).

\section{Availability of data and materials}

Not applicable.

\section{Authors' contributions}


TBZ contributed to the conception and design of the study. TBZ, SJL and CLL were responsible for collection of data and performing the statistical analysis and manuscript preparation. WSL and HZZ were responsible for checking the data. All authors were responsible for drafting the manuscript, read and approved the final version.

\section{Ethics approval and consent to participate}

Not applicable.

\section{Consent for publication}

Not applicable.

\section{Competing interests}

The authors declare that they have no competing interests.

\section{References}

1. Pianta TJ, Buckley NA, Peake PW, Endre ZH. Clinical use of biomarkers for toxicant-induced acute kidney injury. Biomark Med. 2013;7(3):441-56.

2. Liang Y, Zhang D, Li L, Xin T, Zhao Y, Ma R, Du J. Exosomal microRNA-144 from bone marrow-derived mesenchymal stem cells inhibits the progression of non-small cell lung cancer by targeting CCNE1 and CCNE2. Stem Cell Res Ther. 2020;11(1):87.

3. Jasmin. In Vitro Labeling Mesenchymal Stem Cells with Superparamagnetic Iron Oxide Nanoparticles: Efficacy and Cytotoxicity. Methods in molecular biology (Clifton NJ). 2020;2118:23550.

4. Zhu Y, Zhang X, Gu R, Liu X, Wang S, Xia D, Li Z, Lian X, Zhang P, Liu Y, et al. LAMA2 regulates the fate commitment of mesenchymal stem cells via hedgehog signaling. Stem Cell Res Ther. 2020;11(1):135.

5. He J, Jiang YL, Wang Y, Tian XJ, Sun SR. Micro-vesicles from mesenchymal stem cells overexpressing miR-34a inhibit transforming growth factor-beta1-induced epithelial-mesenchymal transition in renal tubular epithelial cells in vitro. Chinese medical journal 2020.

6. Li D, Zhang D, Tang B, Zhou Y, Guo W, Kang Q, Wang Z, Shen L, Wei G, He D: Exosomes from Human Umbilical Cord Mesenchymal Stem Cells Reduce Damage from Oxidative Stress and the EpithelialMesenchymal Transition in Renal Epithelial Cells Exposed to Oxalate and Calcium Oxalate Monohydrate. Stem Cells Int 2019, 2019:6935806.

7. Liu B, Ding F, Hu D, Zhou Y, Long C, Shen L, Zhang Y, Zhang D, Wei G. Human umbilical cord mesenchymal stem cell conditioned medium attenuates renal fibrosis by reducing inflammation and epithelial-to-mesenchymal transition via the TLR4/NF-kappaB signaling pathway in vivo and in vitro. Stem Cell Res Ther. 2018;9(1):7. 
8. Xing L, Song E, Yu CY, Jia XB, Ma J, Sui MS, Wang MA, Gao X. Bone marrow-derived mesenchymal stem cells attenuate tubulointerstitial injury through multiple mechanisms in UUO model. Journal of cellular biochemistry. 2019;120(6):9737-46.

9. Zheng J, Wang Q, Leng W, Sun X, Peng J. Bone marrowderived mesenchymal stem cellconditioned medium attenuates tubulointerstitial fibrosis by inhibiting monocyte mobilization in an irreversible model of unilateral ureteral obstruction. Mol Med Rep. 2018;17(6):7701-7.

10. Anan HH, Zidan RA, Shaheen MA, Abd-El Fattah EA. Therapeutic efficacy of bone marrow derived mesenchymal stromal cells versus losartan on adriamycin-induced renal cortical injury in adult albino rats. Cytotherapy. 2016;18(8):970-84.

11. Bi B, Schmitt R, Israilova M, Nishio H, Cantley LG. Stromal cells protect against acute tubular injury via an endocrine effect. Journal of the American Society of Nephrology: JASN. 2007;18(9):2486-96.

12. Bruno S, Grange C, Deregibus MC, Calogero RA, Saviozzi S, Collino F, Morando L, Busca A, Falda M, Bussolati B, et al. Mesenchymal stem cell-derived microvesicles protect against acute tubular injury. Journal of the American Society of Nephrology: JASN. 2009;20(5):1053-67.

13. Cetinkaya B, Unek G, Kipmen-Korgun D, Koksoy S, Korgun ET. Effects of Human Placental Amnion Derived Mesenchymal Stem Cells on Proliferation and Apoptosis Mechanisms in Chronic Kidney Disease in the Rat. International journal of stem cells. 2019;12(1):151-61.

14. Danjuma L, Mok PL, Higuchi A, Hamat RA, Teh SW, Koh AE, Munusamy MA, Arulselvan P, Rajan M, Nambi A, et al. Modulatory and regenerative potential of transplanted bone marrow-derived mesenchymal stem cells on rifampicin-induced kidney toxicity. Regenerative therapy. 2018;9:10010.

15. Elbaghdady HAM, Alwaili MA, El-Demerdash RS. Regenerative potential of bone marrow mesenchymal stem cells on cadmium chloride-induced hepato-renal injury and testicular dysfunction in sprague dawley rats. Ecotoxicol Environ Saf. 2018;164:41-9.

16. Elhusseini FM, Saad MA, Anber N, Elghannam D, Sobh MA, Alsayed A, El-Dusoky S, Sheashaa H, Abdel-Ghaffar H, Sobh M. Long Term Study of Protective Mechanisms of Human Adipose Derived Mesenchymal Stem Cells on Cisplatin Induced Kidney injury in Sprague-Dawley Rats. Journal of stem cells regenerative medicine. 2016;12(1):36-48.

17. Eliopoulos N, Zhao J, Bouchentouf M, Forner K, Birman E, Yuan S, Boivin MN, Martineau D. Human marrow-derived mesenchymal stromal cells decrease cisplatin renotoxicity in vitro and in vivo and enhance survival of mice post-intraperitoneal injection. American journal of physiology Renal physiology. 2010;299(6):F1288-98.

18. Gad AM, Hassan WA, Fikry EM. Significant curative functions of the mesenchymal stem cells on methotrexate-induced kidney and liver injuries in rats. Journal of biochemical and molecular toxicology 2017, 31(8).

19. Herrera MB, Bussolati B, Bruno S, Fonsato V, Romanazzi GM, Camussi G. Mesenchymal stem cells contribute to the renal repair of acute tubular epithelial injury. Int J Mol Med. 2004;14(6):1035-41. 
20. Kim JH, Park DJ, Yun JC, Jung MH, Yeo HD, Kim HJ, Kim DW, Yang JI, Lee GW, Jeong SH, et al. Human adipose tissue-derived mesenchymal stem cells protect kidneys from cisplatin nephrotoxicity in rats. American journal of physiology Renal physiology. 2012;302(9):F1141-50.

21. Magnasco A, Corselli M, Bertelli R, Ibatici A, Peresi M, Gaggero G, Cappiello V, Chiavarina B, Mattioli G, Gusmano R, et al. Mesenchymal stem cells protective effect in adriamycin model of nephropathy. Cell Transplant. 2008;17(10-11):1157-67.

22. Moustafa FE, Sobh MA, Abouelkheir M, Khater Y, Mahmoud K, Saad MA, Sobh MA. Study of the Effect of Route of Administration of Mesenchymal Stem Cells on Cisplatin-Induced Acute Kidney Injury in Sprague Dawley Rats. International journal of stem cells. 2016;9(1):79-89.

23. Putra A, Pertiwi D, Milla MN, Indrayani UD, Jannah D, Sahariyani M, Trisnadi S, Wibowo JW. Hypoxiapreconditioned MSCs Have Superior Effect in Ameliorating Renal Function on Acute Renal Failure Animal Model. Open access Macedonian journal of medical sciences. 2019;7(3):305-10.

24. Qian H, Yang H, Xu W, Yan Y, Chen Q, Zhu W, Cao H, Yin Q, Zhou H, Mao F, et al. Bone marrow mesenchymal stem cells ameliorate rat acute renal failure by differentiation into renal tubular epithelial-like cells. Int J Mol Med. 2008;22(3):325-32.

25. Rashed LA, Elattar S, Eltablawy N, Ashour H, Mahmoud LM, El-Esawy Y. Mesenchymal stem cells pretreated with melatonin ameliorate kidney functions in a rat model of diabetic nephropathy. Biochemistry cell biology $=$ Biochimie et biologie cellulaire. 2018;96(5):564-71.

26. Sarhan M, El Serougy H, Hussein AM, El-Dosoky M, Sobh MA, Fouad SA, Sobh M, Elhusseini F. Impact of bone-marrow-derived mesenchymal stem cells on adriamycin-induced chronic nephropathy. Can J Physiol Pharmacol. 2014;92(9):733-43.

27. Selim RE, Ahmed HH, Abd-Allah SH, Sabry GM, Hassan RE, Khalil WKB, Abouhashem NS. Mesenchymal Stem Cells: a Promising Therapeutic Tool for Acute Kidney Injury. Appl Biochem Biotechnol. 2019;189(1):284-304.

28. Sun JH, Teng GJ, Ma ZL, Ju SH. In vivo monitoring of magnetically labeled mesenchymal stem cells administered intravascularly in rat acute renal failure. Swiss Med Wkly. 2008;138(27-28):404-12.

29. Zickri MB, Zaghloul S, Farouk M, Fattah MM. Effect of stem cell therapy on adriamycin induced tubulointerstitial injury. International journal of stem cells. 2012;5(2):130-9.

30. Mata-Miranda M, Bernal-Barquero C, Martinez-Cuazitl A, Guerrero-Robles C, Sanchez-Monroy V, RojasLopez M, Vazquez-Zapien G: Nephroprotective Effect of Embryonic Stem Cells Reducing Lipid Peroxidation in Kidney Injury Induced by Cisplatin. Oxid Med Cell Longev 2019, 2019:5420624.

31. Vazquez-Zapien G, Martinez-Cuazitl A, Rangel-Cova L, Camacho-Ibarra A, Mata-Miranda M. Biochemical and histological effects of embryonic stem cells in a mouse model of renal failure. Rom J Morphol Embryol. 2019;60(1):189-94.

32. Minocha E, Sinha R, Jain M, Chaturvedi C, Nityanand S. Amniotic fluid stem cells ameliorate cisplatin-induced acute renal failure through induction of autophagy and inhibition of apoptosis. Stem Cell Res Ther. 2019;10(1):370. 
33. Sun B, Luo X, Yang C, Liu P, Yang Y, Dong X, Yang Z, Xu J, Zhang Y, Li L: Therapeutic Effects of Human Urine-Derived Stem Cells in a Rat Model of Cisplatin-Induced Acute Kidney Injury In Vivo and In Vitro. Stem Cells Int 2019, 2019:8035076.

34. Zhang C, George S, Wu R, Thakker P, Abolbashari M, Kim T, Ko I, Zhang Y, Sun Y, Jackson J, et al. Reno-protection of Urine-derived Stem Cells in A Chronic Kidney Disease Rat Model Induced by Renal Ischemia and Nephrotoxicity. Int J Biol Sci. 2020;16(3):435-46.

35. Sun W, Zhu Q, Yan L, Shao F. Mesenchymal stem cells alleviate acute kidney injury via miR-107mediated regulation of ribosomal protein S19. Ann Transl Med. 2019;7(23):765.

36. Foroutan T, Nafar M, Motamedi E. Intraperitoneal Injection of Graphene Oxide Nanoparticle Accelerates Stem Cell Therapy Effects on Acute Kidney Injury. Stem Cells Cloning. 2020;13:21-32.

37. Zhou T, Liao C, Li HY, Lin W, Lin S, Zhong H. Efficacy of mesenchymal stem cells in animal models of lupus nephritis: a meta-analysis. Stem Cell Res Ther. 2020;11(1):48.

38. Chen Y, Tang X, Li P, Zhou Y, Xue T, Liu J, Yu C: Bone Marrow Derived Mesenchymal Stromal Cells Ameliorate Ischemia/Reperfusion Injury-Induced Acute Kidney Injury in Rats via Secreting Tumor Necrosis Factor-Inducible Gene 6 Protein. BioMed research international 2019, 2019:9845709.

39. Xiu GH, Zhou X, Li XL, Chen XZ, Li BQ, Chen XL, Jin H, Pan XH, Sun J, Ling B. Role of Bone Marrow Mesenchymal Stromal Cells in Attenuating Inflammatory Reaction in Lipopolysaccaride-induced Acute Kidney Injury of Rats Associated with TLR4-NF-kappaB Signaling Pathway Inhibition. Ann Clin Lab Sci. 2018;48(6):743-50.

40. El-Metwaly S, El-Senduny FF, El-Demerdash RS, Abdel-Aziz AF. Mesenchymal stem cells alleviate hydrochloric acid-induced lung injury through suppression of inflammation, oxidative stress and apoptosis in comparison to moxifloxacin and sildenafil. Heliyon. 2019;5(12):e02710.

41. Li P, Chen Y, Yang K, Chen D, Kong D. Mechanical characteristics of BMSCs-intervened sciatic nerve in chronic alcohol-intoxicated animal model. The International journal of neuroscience 2020:1-10.

42. Liu B, Ding FX, Liu Y, Xiong G, Lin T, He DW, Zhang YY, Zhang DY, Wei GH. Human umbilical cordderived mesenchymal stem cells conditioned medium attenuate interstitial fibrosis and stimulate the repair of tubular epithelial cells in an irreversible model of unilateral ureteral obstruction. Nephrology (Carlton Vic). 2018;23(8):728-36.

43. Wang Z, Li S, Wang Y, Zhang X, Chen L, Sun D. GDNF enhances the anti-inflammatory effect of human adipose-derived mesenchymal stem cell-based therapy in renal interstitial fibrosis. Stem cell research. 2019;41:101605.

\section{Figures}


Articles retrieved for review from PubMed, Embase, ISI Web of Science, and Cochrane Library: 785

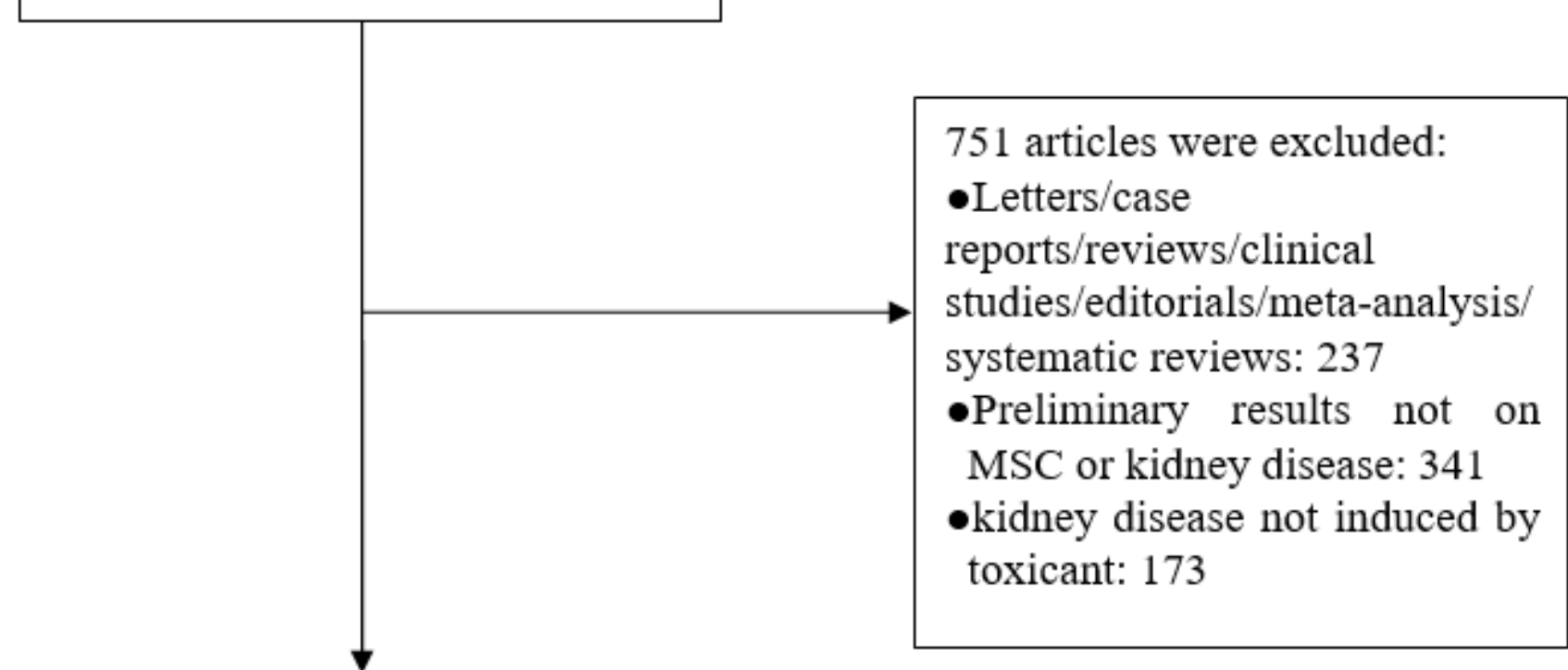

Potentially relevant studies retrieved for more detailed evaluation: 34

$\longrightarrow$\begin{tabular}{l|}
7 studies excluded: \\
-Did not provide the detailed \\
data for case or control group: 3 \\
-Therapeutic regimen for kidney \\
disease including other agents \\
with undefined effects: 4
\end{tabular}

Studies included in the meta-analysis: 27

\section{Figure 1}

Flow diagram of the selection process. 

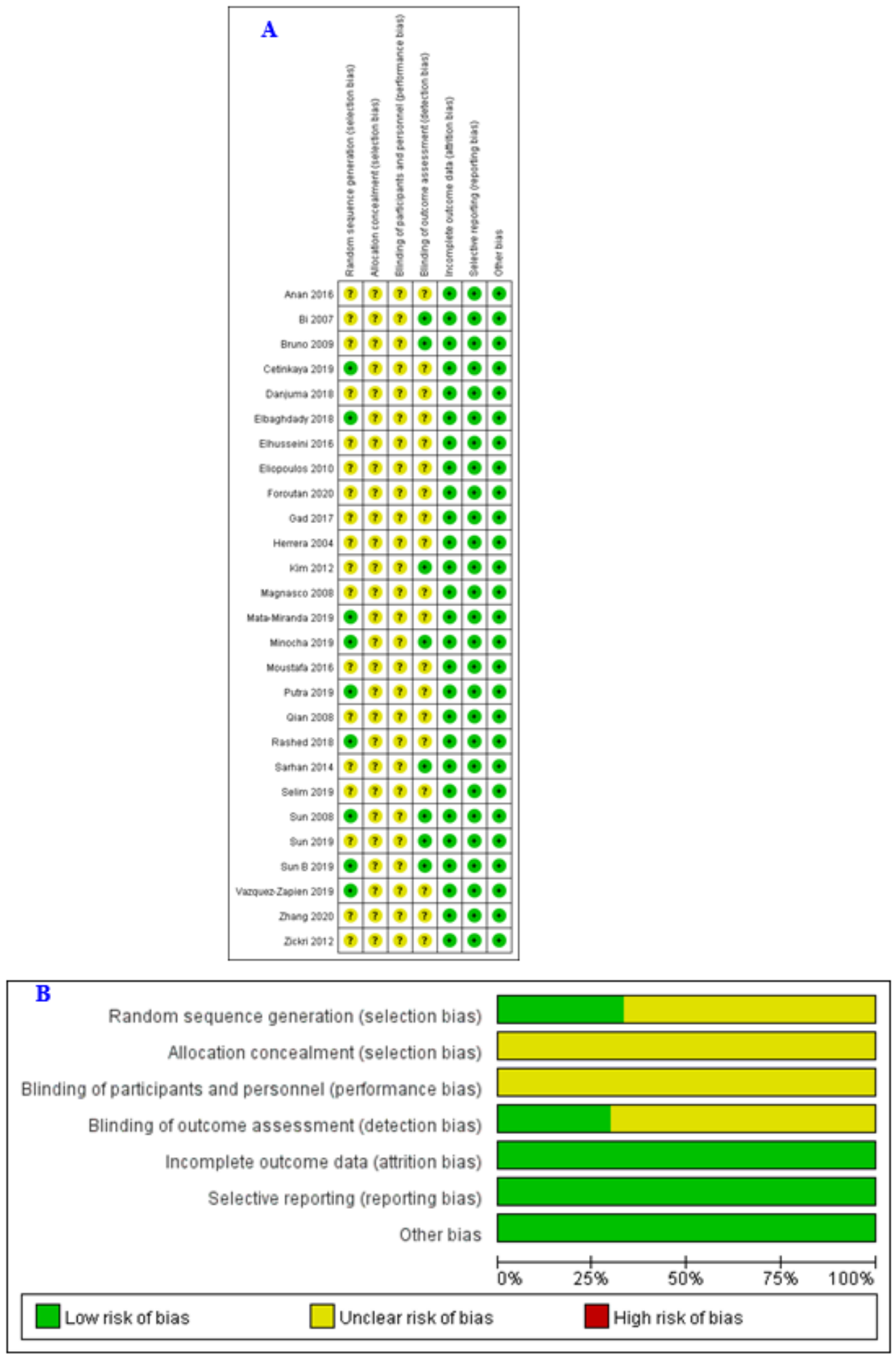

\section{Figure 2}

A: Aggregate Risk of bias graph for each experimental animal studies; B: Risk of bias summary. 


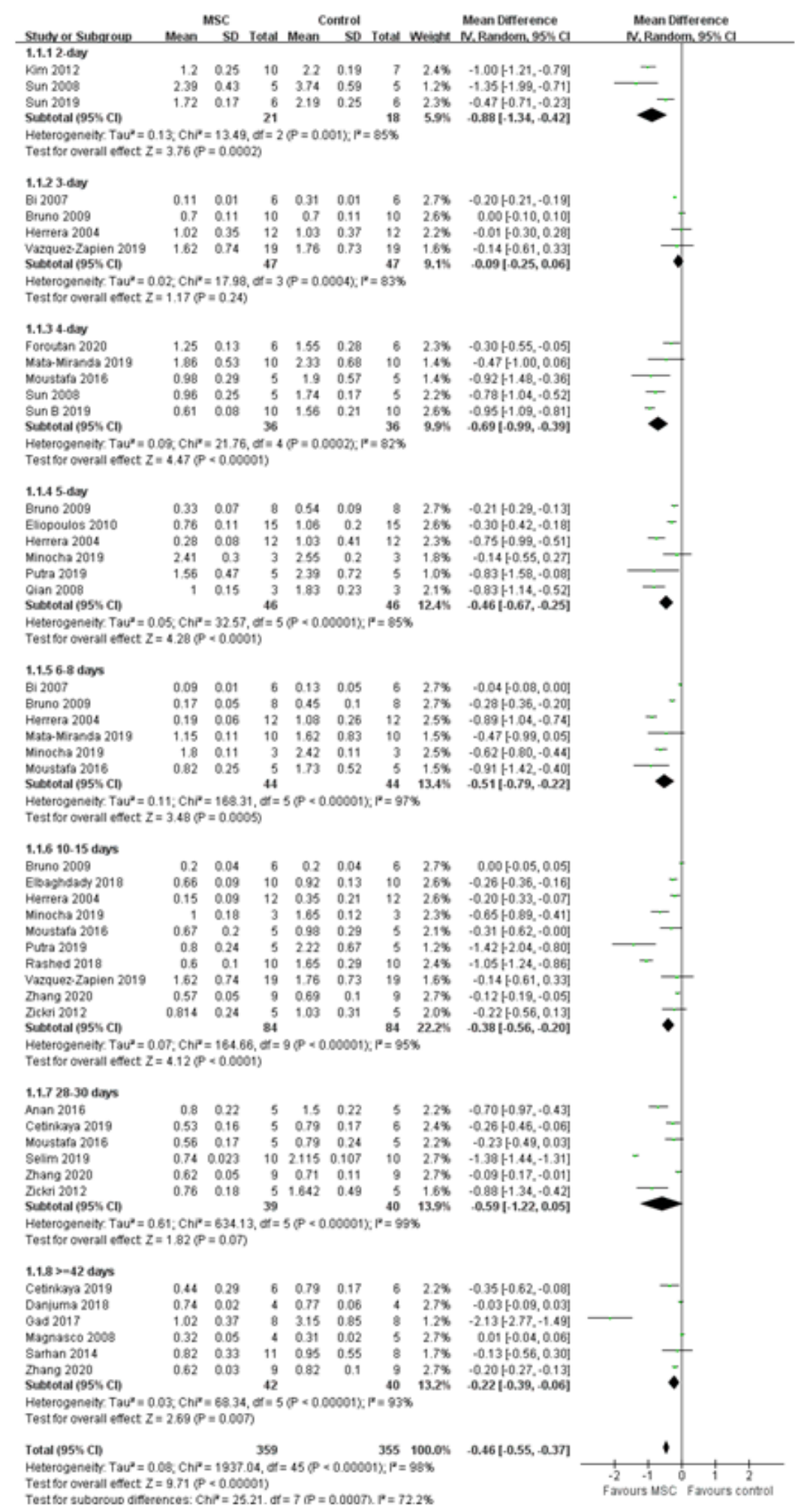

Figure 3

Effect of MSC on Scr. 


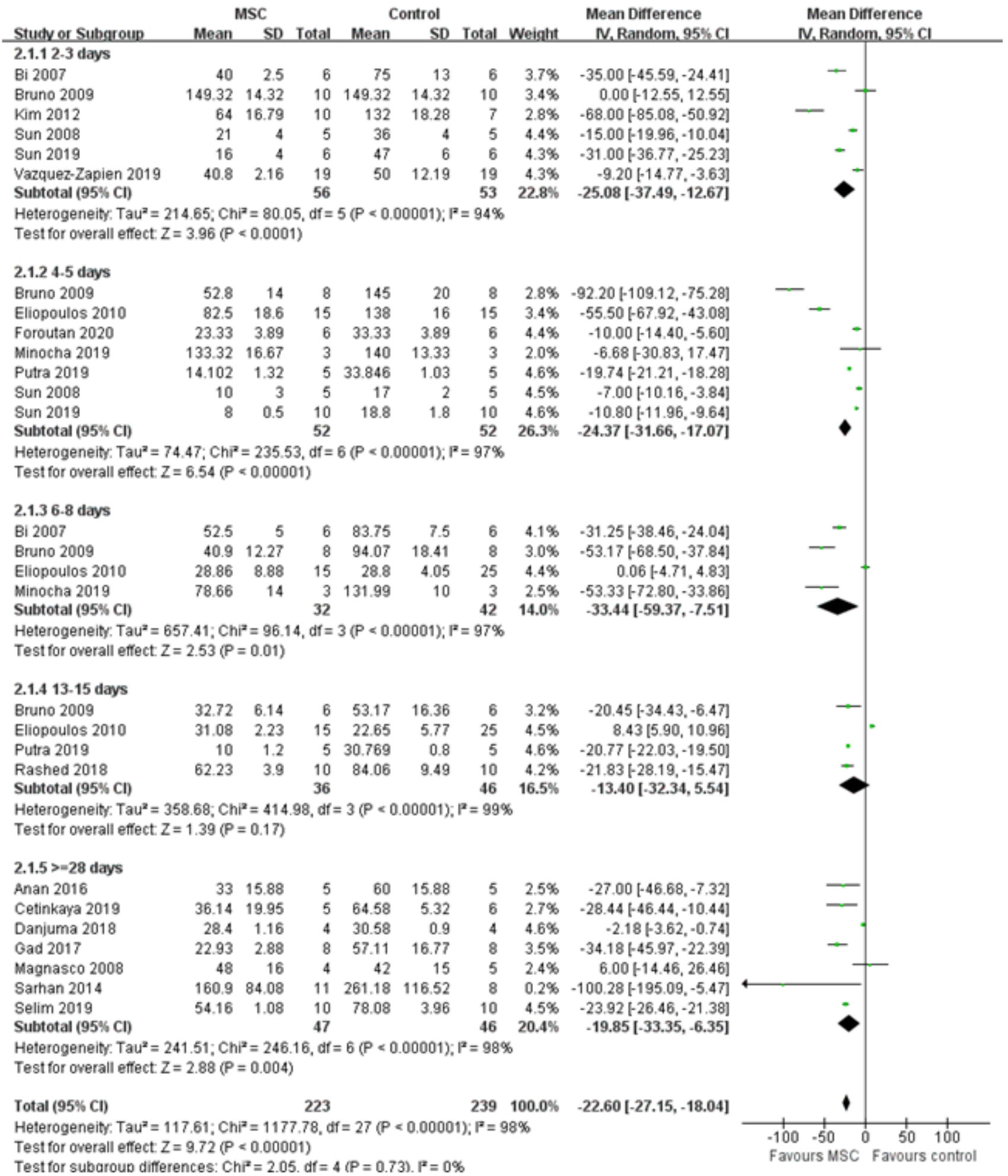

\section{Figure 4}

Effect of MSC on BUN. 
Begg's funnel plot with pseudo $95 \%$ confidence lim its

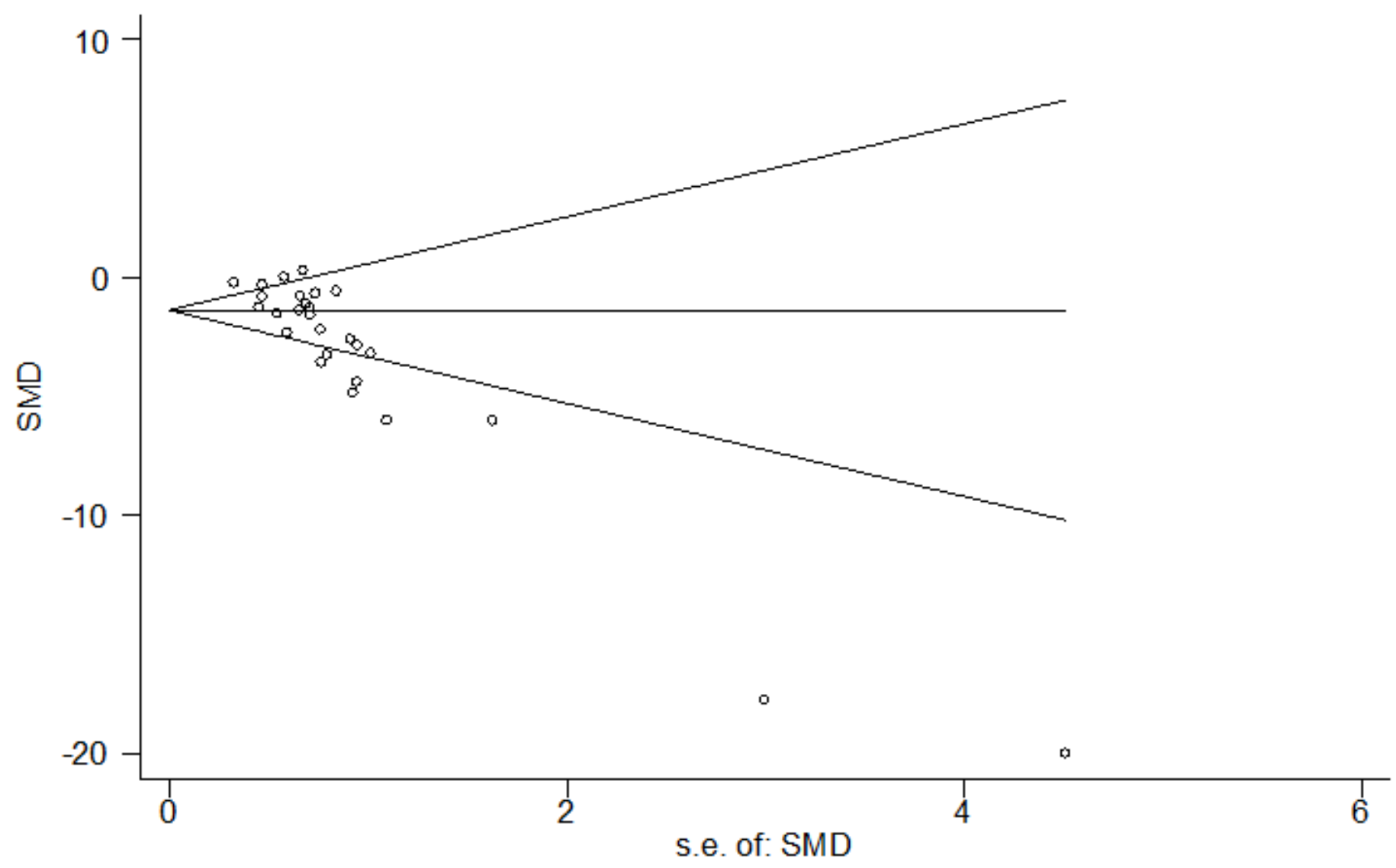

Figure 5

Publication bias. 